\title{
The automorphism group of a free-by-cyclic group in rank 2
}

\author{
O. Bogopolski \\ Institute of Mathematics of SBRAS, \\ Novosibirsk, Russia \\ and Centre de Recerca Matemàtica \\ e-mail: groups@math.nsc.ru \\ A. Martino \\ Dept. Mat. Apl. IV, Univ. Pol. Catalunya, (Barcelona, Spain) \\ and Centre de Recerca Matemàtica \\ e-mail: Armando.Martino@upc.edu \\ E. Ventura \\ Dept. Mat. Apl. III, Univ. Pol. Catalunya, (Barcelona, Spain) \\ and Centre de Recerca Matemàtica \\ e-mail: enric.ventura@upc.edu
}

October 29, 2018

\begin{abstract}
Let $\phi$ be an automorphism of a free group $F_{n}$ of rank $n$, and let $M_{\phi}=F_{n} \rtimes_{\phi} \mathbb{Z}$ be the corresponding mapping torus of $\phi$. We study the group $\operatorname{Out}\left(M_{\phi}\right)$ under certain technical conditions on $\phi$. Moreover, in the case of rank 2, we classify the cases when this group is finite or virtually cyclic, depending on the conjugacy class of the image of $\phi$ in $G L_{2}(\mathbb{Z})$.
\end{abstract}

\section{Introduction}

Let $F_{n}$ be the free group of rank $n$ freely generated by $x_{1}, \ldots, x_{n}$, and let us denote automorphisms $\phi \in \operatorname{Aut}\left(F_{n}\right)$ as acting on the right, $x \mapsto x \phi$. In this paper we consider extensions of finitely generated free groups by the infinite cyclic group ([f.g. free]-by- $\mathbb{Z}$ groups, for short). More precisely, for any given $\phi \in A u t\left(F_{n}\right)$, we consider the mapping torus, $M_{\phi}=F \rtimes_{\phi} \mathbb{Z}$, of $\phi$ i.e. the extension of $F_{n}$ presented by

$$
M_{\phi}=\left\langle x_{1}, \ldots, x_{n}, t \mid t^{-1} x_{i} t=x_{i} \phi(i=1, \ldots, n)\right\rangle .
$$

The aim of the paper is to study the outer automorphism group of such groups, Out $\left(M_{\phi}\right)$. We shall give partial results for arbitrary rank $n$, and a complete description for the cases $n=1,2$.

To help avoiding possible confusions, we will use Greek letters (such as $\phi$ or $\psi$ ) to denote automorphisms of $F_{n}$, and capital Greek letters (such as $\Phi$ or $\Psi$ ) to denote automorphisms of $M_{\phi}$. Accordingly, for every word $w \in F_{n}$, we shall write $\gamma_{w}$ to denote the inner automorphism 
of $F_{n}$ given by right conjugation by $w, x \gamma_{w}=w^{-1} x w$. And, for every element $g \in M_{\phi}$, we shall write $\Gamma_{g}$ to denote the inner automorphism of $M_{\phi}$ given by right conjugation by $g$, $x \Gamma_{g}=g^{-1} x g$. As usual, $\operatorname{Inn}(G)$ denotes the group of inner automorphisms of a group $G$, and $\operatorname{Out}(G)=\operatorname{Aut}(G) / \operatorname{Inn}(G)$.

Although [f.g. free]-by- $\mathbb{Z}$ groups have received a great deal of attention in recent years, there has been no real systematic study of their automorphisms. In fact, it still seems to be an open question whether or not they have finitely generated or finitely presented automorphism groups. Having said that, there are certain cases in which the automorphism group is understood. For instance, when $M_{\phi}$ is word hyperbolic, it is known to have finite outer automorphism group (this can be deduced from papers [1, 3]). However, note that in the rank 2 case, the group $M_{\phi}$ can never be hyperbolic. In fact, by a result of Nielsen (see [6, proposition 5.1]), $\left(\left[x_{1}, x_{2}\right]\right) \phi$ and so $t^{-1}\left[x_{1}, x_{2}\right] t$, must be conjugate to $\left[x_{1}, x_{2}\right]^{ \pm 1}$ in $F_{2}$. Hence, $M_{\phi}$ contains a free abelian subgroup of rank 2 implying that $M_{\phi}$ is not hyperbolic.

The case of rank 1 is straightforward to analyse. If $\phi \in A u t(\mathbb{Z})$ is the identity then $M_{\phi}=$ $F_{1} \rtimes_{\phi} \mathbb{Z}=\mathbb{Z} \times \mathbb{Z}$ and so, Out $\left(M_{\phi}\right)=\operatorname{Aut}\left(M_{\phi}\right)=G L_{2}(\mathbb{Z})$. Otherwise, $\phi$ is the inversion and it is easy to check that $\operatorname{Out}\left(M_{\phi}\right)$ is finite in this case.

The paper is organised as follows. In Section 2, we analyse $A u t\left(M_{\phi}\right)$ for arbitrary $n$ but under certain technical restrictions for $\phi$ (see Theorems 2.4 and 2.5 below). After dedicating Section 3 to recall a standard classification of $2 \times 2$ matrices, the main result comes in Section 4, where we analyse $A u t\left(M_{\phi}\right)$ in the case when the underlying free group has rank $n=2$, and without conditions on $\phi$. The rank 2 case is doubtless the easiest to deal with, but we believe that some of our methods may be of general interest. For instance, our detailed look at the parabolic case is certain to be of use in the more general UPG case (the definition of UPG automorphisms can be found in [2]). The information obtained in Section 4 is summarised in the subsequent theorem, which is the main result of the paper.

Theorem 1.1 Let $F_{2}=\langle a, b\rangle$ be a free group of rank 2, let $\phi \in A u t\left(F_{2}\right)$, and consider the mapping torus $M_{\phi}=F_{2} \rtimes_{\phi} \mathbb{Z}$. Let $\phi^{\mathrm{ab}} \in G L_{2}(\mathbb{Z})$ be the map induced by $\phi$ on $F_{2}^{\mathrm{ab}} \cong \mathbb{Z}^{2}$ (written in row form with respect to $\{a, b\})$.

i) If $\phi^{\mathrm{ab}}=I_{2}$, then $\operatorname{Out}\left(M_{\phi}\right) \cong\left(\mathbb{Z}^{2} \rtimes C_{2}\right) \rtimes G L_{2}(\mathbb{Z})$, where $C_{2}$ is the cyclic group of order 2 acting on $\mathbb{Z}^{2}$ by sending $u$ to $-u, u \in \mathbb{Z}^{2}$, and where $G L_{2}(\mathbb{Z})$ acts trivially on $C_{2}$ and naturally on $\mathbb{Z}^{2}$ (thinking vectors as columns there).

ii) If $\phi^{\mathrm{ab}}=-I_{2}$, then $\operatorname{Out}\left(M_{\phi}\right) \cong P G L_{2}(\mathbb{Z}) \times C_{2}$.

iii) If $\phi^{\mathrm{ab}} \neq-I_{2}$ and does not have 1 as an eigenvalue then Out $\left(M_{\phi}\right)$ is finite.

iv) If $\phi^{\mathrm{ab}}$ is conjugate to $\left(\begin{array}{cc}1 & k \\ 0 & -1\end{array}\right)$ for some integer $k$, then Out $\left(M_{\phi}\right)$ has an infinite cyclic subgroup of finite index.

v) If $\phi^{\mathrm{ab}}$ is conjugate to $\left(\begin{array}{ll}1 & k \\ 0 & 1\end{array}\right)$ for some integer $k \neq 0$, then Out $\left(M_{\phi}\right)$ has an infinite cyclic subgroup of finite index.

Furthermore, for every $\phi \in A u t\left(F_{2}\right), \phi^{\mathrm{ab}}$ fits into exactly one of the above cases.

We note that in rank $2, M_{\phi}$ is always the fundamental group of a 3-manifold, namely the corresponding mapping torus of a once punctured torus, say $M$. When $\phi^{\text {ab }}$ is hyperbolic, $M$ is a hyperbolic manifold and it is known that, in this case, by Mostow rigidity, Out $\left(\pi_{1} M\right)=\operatorname{Out}\left(M_{\phi}\right)$ 
is finite. This fact is contained in Theorem 1.1 (iii), which we prove by elementary methods. Although this is the generic case, we believe there is value in analysing the entire situation, especially since the proof of Theorem 1.1 (v) is rather more involved than one might expect.

We remark that the isomorphism type of $M_{\phi}$ depends only on the conjugacy class of the outer automorphism $[\phi]^{ \pm 1} \in \operatorname{Out}\left(F_{n}\right)=\operatorname{Aut}\left(F_{n}\right) / \operatorname{Inn}\left(F_{n}\right)$ determined by $\phi^{ \pm 1}$ (this is straightforward to verify, see Lemma 2.1 below). As an application of our main result, we prove that this characterises the isomorphism class of $M_{\phi}$, when $n=2$.

Corollary 1.2 Let $F_{2}=\langle a, b\rangle$ be a free group of rank 2 and let $\phi, \psi \in \operatorname{Aut}\left(F_{2}\right)$. The groups $M_{\phi}$ and $M_{\psi}$ are isomorphic if and only if $[\phi]$ and $[\psi]^{ \pm 1}$ are conjugate in Out $\left(F_{2}\right)$.

Proof. The if part is straightforward and proven in Lemma 2.1 below.

Let us assume $M_{\phi} \cong M_{\psi}$ (and so, $\left.\operatorname{Out}\left(M_{\phi}\right) \cong \operatorname{Out}\left(M_{\psi}\right)\right)$. By Theorem 1.1. $\phi$ and $\psi$ fit each one into exactly one of the items (i) to (v). But the groups $\left(\mathbb{Z}^{2} \rtimes C_{2}\right) \rtimes G L_{2}(\mathbb{Z}), P G L_{2}(\mathbb{Z}) \times C_{2}$, a finite group, and a virtually- $\mathbb{Z}$ group are not pairwise isomorphic (note, for example, that the first one contains $\mathbb{Z}^{2}$ and the second one is virtually free). So, $\phi$ and $\psi$ fit simultaneously into case (i), or case (ii), or case (iii), or cases (iv)-(v). In order to distinguish also between cases (iv) and (v), note that $\left(\begin{array}{cc}1 & k \\ 0 & -1\end{array}\right)^{2}=\left(\begin{array}{ll}1 & 0 \\ 0 & 1\end{array}\right)$ while $\left(\begin{array}{ll}1 & k \\ 0 & 1\end{array}\right)$ has infinite order; Lemma 2.2 then, says that $M_{\phi} \cong M_{\psi}$ has non-trivial and trivial centre, respectively.

Suppose $\phi$ and $\psi$ both fit into case (i) or both into case (ii). Then $\phi^{\mathrm{ab}}=\psi^{\mathrm{ab}}$, and so $\phi \psi^{-1}$ is an inner automorphism of $F_{2}$. Thus, $[\phi]=[\psi]$ in $\operatorname{Out}\left(F_{2}\right)$.

Suppose $\phi$ and $\psi$ both fit into case (iii) and so, $\phi^{\text {ab }}$ and $\psi^{\text {ab }}$ do not have 1 as an eigenvalue. By Theorem 2.4, $F_{2}$ is the unique normal subgroup of $M_{\phi}$ and of $M_{\psi}$ with quotient isomorphic to $\mathbb{Z}$. Hence, the given isomorphism $\Xi: M_{\phi} \rightarrow M_{\psi}$ must preserve the free part, $F_{2}$, and so must send $t$ to $t^{\epsilon} w$, for some $\epsilon= \pm 1$ and $w \in F_{2}$. Let $\xi \in A u t\left(F_{2}\right)$ be the restriction of $\Xi$ to $F_{2}$. For every $x \in F_{2}$, the relation $t^{-1} x t=x \phi$ (valid in $M_{\phi}$ ) implies $w^{-1}\left(x \xi \psi^{\epsilon}\right) w=w^{-1} t^{-\epsilon}(x \xi) t^{\epsilon} w=x \phi \xi$ in $M_{\psi}$. So, we have $\xi \psi^{\epsilon} \gamma_{w}=\phi \xi$ in $\operatorname{Aut}\left(F_{2}\right)$, which means that $[\phi]$ and $[\psi]^{\epsilon}$ are conjugate in $\operatorname{Out}\left(F_{2}\right)$.

Suppose $\phi$ and $\psi$ both fit into case (iv), say $\phi^{\text {ab }}$ is conjugate to $\left(\begin{array}{ll}1 & k_{1} \\ 0 & -1\end{array}\right)$ and $\psi^{\text {ab }}$ is conjugate to $\left(\begin{array}{ll}1 & k_{2} \\ 0 & -1\end{array}\right)$. It is straightforward to verify that these two matrices are conjugate to each other in $G L_{2}(\mathbb{Z})$ if and only if $k_{1}$ and $k_{2}$ have the same parity. So, $\phi^{\text {ab }}$ (and similarly $\psi^{\text {ab }}$ ) is conjugate to either $\left(\begin{array}{cc}1 & 0 \\ 0 & -1\end{array}\right)$ or $\left(\begin{array}{cc}1 & 1 \\ 0 & -1\end{array}\right)$. Accordingly, $M_{\phi}\left(\right.$ and $\left.M_{\psi}\right)$ is isomorphic to either

$$
M_{\left(\begin{array}{cc}
1 & 0 \\
0 & -1
\end{array}\right)}=\left\langle a, b, t \mid t^{-1} a t=a, t^{-1} b t=b^{-1}\right\rangle
$$

or

$$
M_{\left(\begin{array}{cc}
1 & 1 \\
0 & -1
\end{array}\right)}=\left\langle a, b, t \mid t^{-1} a t=a b, t^{-1} b t=b^{-1}\right\rangle .
$$

But, looking at their abelianisations, these two groups are not isomorphic to each other (the first abelianises to $\mathbb{Z}^{2} \oplus \mathbb{Z} / 2 \mathbb{Z}$ and the second to $\mathbb{Z}^{2}$ ). Thus, $k_{1}$ and $k_{2}$ have the same parity and so, $[\phi]$ and $[\psi]$ are conjugate in $\operatorname{Out}\left(F_{2}\right)$.

Finally, suppose $\phi$ and $\psi$ both fit into case (v), say $\phi^{\text {ab }}$ is conjugate to $\left(\begin{array}{cc}1 & k_{1} \\ 0 & 1\end{array}\right)$ and $\psi^{\text {ab }}$ is conjugate to $\left(\begin{array}{cc}1 & k_{2} \\ 0 & 1\end{array}\right)$. Easy computations show that $M_{\phi}^{\text {ab }} \cong \mathbb{Z}^{2} \oplus \mathbb{Z} /\left|k_{1}\right| \mathbb{Z}$ and $M_{\psi}^{\text {ab }} \cong \mathbb{Z}^{2} \oplus \mathbb{Z} /\left|k_{2}\right| \mathbb{Z}$. Hence, $k_{1}= \pm k_{2}$. And, for every $k$, the matrix $\left(\begin{array}{cc}1 & 0 \\ 0 & -1\end{array}\right)$ conjugates $\left(\begin{array}{ll}1 & k \\ 0 & 1\end{array}\right)$ into $\left(\begin{array}{cc}1 & -k \\ 0 & 1\end{array}\right)$. So, $[\phi]$ and $[\psi]$ are conjugate in $\operatorname{Out}\left(F_{2}\right)$. 
Remark 1.3 We remark that some results similar to Corollary 1.2 were already known in the abelian context. For example, in the first appendix of [4] the authors show that two $\mathbb{Z}^{2}$-by-cyclic groups are isomorphic if and only if the corresponding actions (i.e. matrices in $G L_{2}(\mathbb{Z})$ ) are conjugated one to the other or one to the inverse of the other (our Corollary 1.2 states exactly the same changing $\mathbb{Z}^{2}$ to $F_{2}$ ). Also, W. Dicks extended that result to dimension 3 with the extra hypothesis that the corresponding $3 \times 3$ matrices have no non-trivial eigenvectors of eigenvalue one (see section 4.5 of [5]).

For completeness, let us emphasise that Corollary 1.2 is no longer true for general rank. However, finding two explicit non-conjugate (neither inverse-conjugate) outer automorphisms $\phi$ and $\psi$ of a free group such that $M_{\phi} \cong M_{\psi}$ does not seem to be straightforward. We present the following example, which is the simplest in a series of examples we have found. We thank Joan Porti for pointing out to us certain manifolds that allowed us to construct an explicit example in rank 6 . We also thank Warren Dicks, who then provided a rank 3 example, and allowed us to include it here. Also, we thank R. Vikent'ev (Bogopolski's student) for proposing some other similar examples in rank 3.

Dicks' example is the following. Consider the group presented by

$$
G=\left\langle s, t \mid t^{-3} s t^{2} s t^{-1} s^{-1} t s^{-2} t s\right\rangle
$$

On one hand we have

$$
\begin{aligned}
G & \cong\left\langle s_{0}, s_{1}, s_{2}, s_{3}, t \mid s_{1}=t^{-1} s_{0} t, s_{2}=t^{-1} s_{1} t, s_{3}=t^{-1} s_{2} t, s_{3} s_{1} s_{2}^{-1} s_{1}^{-2} s_{0}=1\right\rangle \\
& \cong\left\langle s_{0}, s_{1}, s_{2}, t \mid t^{-1} s_{0} t=s_{1}, t^{-1} s_{1} t=s_{2}, t^{-1} s_{2} t=s_{0}^{-1} s_{1}^{2} s_{2} s_{1}^{-1}\right\rangle \\
& \cong M_{\phi},
\end{aligned}
$$

where $\phi$ is the automorphism of $F_{3}=\langle a, b, c\rangle$ given by $a \rightarrow b \rightarrow c \rightarrow a^{-1} b^{2} c b^{-1}$. On the other hand,

$$
\begin{aligned}
G & \cong\left\langle t_{-2}, t_{-1}, t_{0}, t_{1}, s \mid t_{-1}=s^{-1} t_{-2} s, t_{0}=s^{-1} t_{-1} s, t_{1}=s^{-1} t_{0} s, t_{0}^{-3} t_{-1}^{2} t_{-2}^{-1} t_{-1} t_{1}=1\right\rangle \\
& \cong\left\langle t_{-2}, t_{-1}, t_{0}, s \mid s^{-1} t_{-2} s=t_{-1}, s^{-1} t_{-1} s=t_{0}, s^{-1} t_{0} s=t_{-1}^{-1} t_{-2} t_{-1}^{-2} t_{0}^{3}\right\rangle \\
& \cong M_{\psi},
\end{aligned}
$$

where $\psi$ is the automorphism of $F_{3}=\langle a, b, c\rangle$ given by $a \rightarrow b \rightarrow c \rightarrow b^{-1} a b^{-2} c^{3}$.

This proves that $M_{\phi} \cong M_{\psi}$, while the outer automorphisms $[\phi],[\psi] \in \operatorname{Out}\left(F_{3}\right)$ are neither conjugate nor inverse-conjugate since their abelianisations are two $3 \times 3$ matrices with determinant -1 and 1 , respectively. This concludes the desired example.

\section{Results for general rank}

Using the defining relations of $M_{\phi}$ under the form $w t=t(w \phi)$ and $w t^{-1}=t^{-1}\left(w \phi^{-1}\right), w \in F_{n}$, it is clear that in any element of $M_{\phi}$ we can push all the $t$ letters to one side. That is, we have a normal form in $M_{\phi}$ whereby we can write elements uniquely in the form $t^{k} w$, where $k$ is an integer and $w \in F_{n}$.

Our first observation is that the isomorphism type of $M_{\phi}$ depends only on the outer automorphism determined by $\phi^{ \pm 1}$, up to conjugacy in $\operatorname{Out}\left(F_{n}\right)$.

Lemma 2.1 Let $F_{n}$ be a free group of rank $n$, let $\phi, \psi \in A u t\left(F_{n}\right)$ and consider

$$
M_{\phi}=\left\langle x_{1}, \ldots, x_{n}, t \mid t^{-1} x_{i} t=x_{i} \phi(i=1, \ldots, n)\right\rangle
$$


and

$$
M_{\psi}=\left\langle x_{1}, \ldots, x_{n}, s \mid s^{-1} x_{i} s=x_{i} \psi(i=1, \ldots, n)\right\rangle .
$$

If the automorphisms $\phi$ and $\psi$ are conjugate or conjugate-inverse to each other in Out $\left(F_{n}\right)$, then $M_{\phi}$ and $M_{\psi}$ are isomorphic. More precisely, if $\chi \in \operatorname{Aut}\left(F_{n}\right)$ is such that $\chi^{-1} \phi \chi=\psi^{\epsilon} \gamma_{w}$ for some $\epsilon= \pm 1$ and $w \in F_{n}$, then the map $\Omega: M_{\phi} \rightarrow M_{\psi}, x_{i} \mapsto x_{i} \chi, t \mapsto s^{\epsilon} w$ extends to an isomorphism.

Proof. The map $\Omega$ is extended multiplicatively and one needs to show that it is well defined. In order to do this, it is enough to show that the relators in $M_{\phi}$ are all sent to the trivial element in $M_{\psi}$. In fact, for every $i=1, \ldots, n$, we have

$$
\begin{aligned}
t^{-1} x_{i} t\left(x_{i} \phi\right)^{-1} & \mapsto w^{-1} s^{-\epsilon}\left(x_{i} \chi\right) s^{\epsilon} w\left(x_{i} \phi \chi\right)^{-1} \\
& =w^{-1}\left(x_{i} \chi \psi^{\epsilon}\right) w\left(x_{i} \phi \chi\right)^{-1} \\
& =\left(x_{i} \chi \psi^{\epsilon} \gamma_{w}\right)\left(x_{i} \phi \chi\right)^{-1} \\
& =\left(x_{i} \phi \chi\right)\left(x_{i} \phi \chi\right)^{-1} \\
& =1 \in M_{\psi}
\end{aligned}
$$

Thus, we have a well defined homomorphism $\Omega: M_{\phi} \rightarrow M_{\psi}$, which is surjective by inspection. Moreover, if an element $t^{k} x \in M_{\phi}$ is in the kernel of $\Omega$, we immediately deduce that $k=0$ and $x \chi=1$. Since $\chi$ is an automorphism of $F_{n}$, we have $x=1$ and $\Omega$ has trivial kernel.

We continue with some basic facts about the automorphism group of $M_{\phi}$.

Lemma 2.2 Let $F_{n}$ be a free group of rank $n$, and let $\phi \in \operatorname{Aut}\left(F_{n}\right)$. The group $M_{\phi}=F_{n} \rtimes_{\phi} \mathbb{Z}$ has non-trivial centre if and only if $\phi^{k}=\gamma_{w}$ for some $k \neq 0$ and some $w \in F_{n}$. If this equation holds and $n \geqslant 2$, then $w \phi=w$.

Proof. The result is clear for $n=0,1$. So, we may assume that $n \geqslant 2$.

A straightforward calculation shows that the element $t^{k} w^{-1} \in M_{\phi}$ commutes with every $x \in F_{n}$ if and only if $\phi^{k}=\gamma_{w}$. Similarly, $t^{k} w^{-1}$ commutes with $t$ if and only if $w \phi=w$. So, $t^{k} w^{-1}$ is central in $M_{\phi}$ if and only if $\phi^{k}=\gamma_{w}$ and $w \phi=w$. Hence, $M_{\phi}$ has non-trivial centre if and only if $\phi^{k}=\gamma_{w}$ and $w \phi=w$ for some integer $k$ and some $w \in F_{n}$ such that $(k, w) \neq(0,1)$.

Now, using the fact $n \geqslant 2$, we can simplify this. Assume the equation $\phi^{k}=\gamma_{w}$ holds. Since, $\gamma_{w}=\phi^{-1} \gamma_{w} \phi=\gamma_{w \phi}$ we have $w \phi=w$. Also, note that $k=0$ implies $w=1$ (because $F_{n}$ has trivial centre for $n \geqslant 2$ ). Thus, $M_{\phi}$ has non-trivial centre if and only if $\phi^{k}=\gamma_{w}$ for some integer $k \neq 0$ and some $w \in F_{n}$.

Let $\Psi \in A u t\left(M_{\phi}\right)$ and suppose it leaves $F_{n}$ invariant. In this situation, its restriction to $F_{n}$, $\psi=\left.\Psi\right|_{F_{n}}$, is an endomorphism of $F_{n}$ that will be seen in the next proposition to be always an automorphism. On the other hand, factorising by the normal and $\Psi$-invariant subgroup $F_{n}$, we get an automorphism $\bar{\Psi}$ of $M_{\phi} / F_{n} \cong \mathbb{Z}$. If $\bar{\Psi}=I d$ we shall say that $\Psi$ is a positive automorphism of $M_{\phi}$. Otherwise, $\bar{\Psi}$ is the inversion of $\mathbb{Z}$ and we say that $\Psi$ is negative. In any case, $t \Psi=t^{\epsilon} w$ for some $w \in F$, where $\epsilon= \pm 1$ is the signum of $\Psi$.

Proposition 2.3 Let $F_{n}$ be a free group of rank $n$, let $\phi \in A u t\left(F_{n}\right)$ and consider $M_{\phi}=F_{n} \rtimes_{\phi} \mathbb{Z}$. Let $\Psi \in \operatorname{Aut}\left(M_{\phi}\right)$ be such that $F_{n} \Psi \leqslant F_{n}$, and denote by $\psi: F_{n} \rightarrow F_{n}$ its restriction to $F_{n}$. Then,

i) $\psi$ is an automorphism of $F_{n}$, 
ii) there exists $w \in F_{n}$ such that $\phi \psi=\psi \phi^{\epsilon} \gamma_{w}$, where $\epsilon$ is the signum of $\Psi$. Furthermore, if $n \geqslant 2$ then the word $w$ is unique and satisfies the equation $t \Psi=t^{\epsilon} w$.

Proof. Since $F_{n} \Psi \leqslant F_{n}$, we must have that $t \Psi=t^{ \pm 1} w$ for some $w \in F_{n}$ (otherwise, $t$ would not be in the image of $\Psi)$. Now, clearly, $F_{n} \Psi$ is a normal subgroup of $M_{\phi}=M_{\phi} \Psi=\left\langle F_{n} \psi, t^{ \pm 1} w\right\rangle$. Hence any element $g \in M_{\phi}$ can be written in the form $g=(v \psi)\left(t^{ \pm 1} w\right)^{k}$ for some $v \in F_{n}$ and $k \in \mathbb{Z}$. And here $g \in F_{n}$ if and only if $k=0$. Thus, $F_{n} \Psi=F_{n}$ and $\Psi$ induces an automorphism on $F_{n}$. This proves (i).

Let $\epsilon= \pm 1$ be the signum of $\Psi$, that is, $t \Psi=t^{\epsilon} w$ for some $w \in F_{n}$. Applying $\Psi$ to both sides of the equality $x \phi=t^{-1} x t$ we get

$$
x \phi \psi=w^{-1} t^{-\epsilon}(x \psi) t^{\epsilon} w=x \psi \phi^{\epsilon} \gamma_{w},
$$

for all $x \in F_{n}$. Hence, $\phi \psi=\psi \phi^{\epsilon} \gamma_{w}$. Furthermore, if $n \geqslant 2$, this last equation can only hold for a unique $w \in F_{n}$. This proves (ii).

In the following result, we impose certain hypothesis on $\phi$ to ensure that every automorphism of $M_{\phi}$ leaves the free subgroup invariant. Under these circumstances, computing $\operatorname{Out}\left(M_{\phi}\right)$ is fairly straightforward.

Theorem 2.4 Let $F_{n}$ be a free group of rank $n$, let $\phi \in A u t\left(F_{n}\right)$ and consider $M_{\phi}=F_{n} \rtimes_{\phi} \mathbb{Z}$. Let $M_{\phi}^{\mathrm{ab}}$ denote the abelianisation of $M_{\phi}$, and $F_{n}^{\mathrm{ab}} \cong \mathbb{Z}^{n}$ the abelianisation of $F_{n}$ (which is not in general the image of $F_{n} \leqslant M_{\phi}$ in $\left.M_{\phi}^{\mathrm{ab}}\right)$. Let $\phi^{\mathrm{ab}} \in G L_{n}(\mathbb{Z})$ be the map induced by $\phi$ on $F_{n}^{\mathrm{ab}}$, and $[\phi]$ be the class of $\phi$ in $\operatorname{Out}\left(F_{n}\right)$. The following are equivalent:

a) $M_{\phi}^{\mathrm{ab}}$ is the direct sum of an infinite cyclic group and a finite abelian group,

b) the matrix $\phi^{\mathrm{ab}}$ does not have eigenvalue 1 .

c) $F_{n} \leqslant M_{\phi}$ is the unique normal subgroup of $M_{\phi}$ with quotient isomorphic to $\mathbb{Z}$.

Furthermore, if these conditions hold then every automorphism of $M_{\phi}$ leaves $F_{n}$ invariant,

$$
\text { Aut }^{+}\left(M_{\phi}\right)=\left\{\Psi \in \operatorname{Aut}\left(M_{\phi}\right) \mid \Psi \text { is positive }\right\}
$$

is a normal subgroup of Aut $\left(M_{\phi}\right)$ of index at most 2 and moreover, if $n \geqslant 2$, its image Out ${ }^{+}\left(M_{\phi}\right)$ in Out $\left(M_{\phi}\right)$ is also normal, of index at most two, and isomorphic to $C([\phi]) /\langle[\phi]\rangle$, where $C([\phi])$ denotes the centraliser of $[\phi]$ in $\operatorname{Out}\left(F_{n}\right)$.

Proof. To prove the equivalence of (a) and (b), note that $M_{\phi}^{\mathrm{ab}} \cong\langle t \mid\rangle \oplus F_{n}^{\mathrm{ab}} / \operatorname{Im}\left(\phi^{\mathrm{ab}}-\operatorname{Id}\right)$. Then, $F_{n}^{\mathrm{ab}} / \operatorname{Im}\left(\phi^{\mathrm{ab}}-I d\right)$ is finite if and only if $\operatorname{rank}_{\mathbb{Z}}\left(\operatorname{Im}\left(\phi^{\mathrm{ab}}-I d\right)\right)=\operatorname{rank}_{\mathbb{Z}}\left(F_{n}^{\mathrm{ab}}\right)=n$. And this happens if and only if $\operatorname{rank}_{\mathbb{Z}}\left(\operatorname{Ker}\left(\phi^{\mathrm{ab}}-I d\right)\right)=0$, which is the same as saying that $\phi^{\mathrm{ab}}$ has no eigenvalue 1 . On the other hand, every epimorphism from $M_{\phi}$ onto $\mathbb{Z}$ factors through $M_{\phi}^{\text {ab }}$. So, the equivalence between (a) and (c) is clear $\left(F_{n}\right.$ being the preimage in $M_{\phi}$ of the torsion subgroup of $\left.M_{\phi}^{\mathrm{ab}}\right)$.

We shall now prove the remaining assertions under the assumption that these three conditions hold. Consider the abelianisation map $M_{\phi} \rightarrow M_{\phi}^{\mathrm{ab}}$. Since $F_{n}^{\mathrm{ab}} / \operatorname{Im}\left(\phi^{\mathrm{ab}}-I d\right)$ is the torsion subgroup of $M_{\phi}^{\mathrm{ab}}$, its full pre-image $F_{n}$ is characteristic in $M_{\phi}$. Hence, every automorphism of $M_{\phi}$ leaves $F_{n}$ invariant. At this point, it is clear that $A u t^{+}\left(M_{\phi}\right)$ is a normal subgroup of $\operatorname{Aut}\left(M_{\phi}\right)$ of index at most 2 , and so is $\mathrm{Out}^{+}\left(M_{\phi}\right)$ in $\operatorname{Out}\left(M_{\phi}\right)$. 
Assuming $n \geqslant 2$, it remains to prove that $\operatorname{Out}^{+}\left(M_{\phi}\right) \cong C([\phi]) /\langle[\phi]\rangle$. Define the map

$$
\begin{array}{cl}
f: \operatorname{Aut}^{+}\left(M_{\phi}\right) & \rightarrow C([\phi]) /\langle[\phi]\rangle \\
\Psi & \mapsto\left[\left.\Psi\right|_{F_{n}}\right]\langle[\phi]\rangle .
\end{array}
$$

Note that by Proposition [2.3, the image of this map lies in $C([\phi]) /\langle[\phi]\rangle$. Clearly, $f$ is a homomorphism.

First, we will prove that $\operatorname{Im} f=C([\phi]) /\langle[\phi]\rangle$. Let $\psi \in \operatorname{Aut}\left(F_{n}\right)$ be such that $[\psi] \in C([\phi])$. Then, $\phi \psi=\psi \phi \gamma_{w}$ for some $w \in F_{n}$. In this situation, it is straightforward to verify that $\psi$ extends to a well defined automorphism $\Psi$ of $M_{\phi}$ by just sending $t$ to $t w$. Clearly, $\Psi \in A u t^{+}\left(M_{\phi}\right)$ and its $f$-image is $[\psi]\langle[\phi]\rangle$.

Now we will prove that $\operatorname{Ker} f=\operatorname{Inn}\left(M_{\phi}\right)$. For every element $g=t^{k} w \in M_{\phi}$, we have that $\left.\Gamma_{g}\right|_{F_{n}}=\phi^{k} \gamma_{w}$ and so, $\Gamma_{g}$ maps under $f$ to the identity element of $C([\phi]) /\langle[\phi]\rangle$. This means that $\operatorname{Ker} f \geqslant \operatorname{Inn}\left(M_{\phi}\right)$. Conversely, let $\Psi \in \operatorname{Ker} f$. Then, $\left.\Psi\right|_{F_{n}}=\phi^{k} \gamma_{w}=\left.\Gamma_{t^{k} w}\right|_{F_{n}}$ for some integer $k$ and some $w \in F_{n}$. Also, applying $\Psi$ to both sides of the equation $t^{-1} x t=x \phi$ and using the positivity of $\Psi$ and the fact $n \geqslant 2$, it is straightforward to check that $t \Psi=t(w \phi)^{-1} w=t \Gamma_{t^{k}} w$. Thus, $\Psi=\Gamma_{t^{k} w}$. This completes the proof that $\operatorname{Ker} f=\operatorname{Inn}\left(M_{\phi}\right)$ and so, Out $^{+}\left(M_{\phi}\right) \cong$ $C([\phi]) /\langle[\phi]\rangle$.

The extreme opposite case to the one considered above is when $\phi$ is the identity automorphism (or, in fact, an inner automorphism). We also calculate the automorphism group in this case.

Theorem 2.5 Let $F_{n}=\left\langle x_{1}, \ldots, x_{n}\right\rangle$ be a free group of rank $n \geqslant 2$, and let $M=M_{I d}=F_{n} \times \mathbb{Z}$. Consider the group $\mathbb{Z}^{n} \rtimes C_{2}$ where $C_{2}$ is the cyclic group of order 2 which acts by sending $u$ to $-u$ for all $u \in \mathbb{Z}^{n}$ (think $u$ as a column vector); also, consider the action of $A u t\left(F_{n}\right)$ (and also Out $\left(F_{n}\right)$ ) on it given by the trivial action on $C_{2}$, and the natural action after abelianisation on $\mathbb{Z}^{n}$. Then, Aut $(M) \cong\left(\mathbb{Z}^{n} \rtimes C_{2}\right) \rtimes \operatorname{Aut}\left(F_{n}\right)$ and $\operatorname{Out}(M) \cong\left(\mathbb{Z}^{n} \rtimes C_{2}\right) \rtimes \operatorname{Out}\left(F_{n}\right)$.

Proof. Clearly, distinct automorphisms of $F_{n}$ extend to distinct positive automorphisms of $M$ by sending $t$ to $t$. In this sense, we shall think $A u t\left(F_{n}\right)$ as a subgroup of $A u t(M)$. On the other hand, consider $\mathbb{Z}^{n} \rtimes C_{2}=\mathbb{Z}^{n} \rtimes\langle v\rangle$ so that $v^{-1} u v=-u$ for all $u \in \mathbb{Z}^{n}$. It is straightforward to verify that this group acts faithfully on $M=F_{n} \times \mathbb{Z}$, whereby an element $\left(v^{\epsilon}, u\right), u=\left(u_{1}, \ldots, u_{n}\right)^{T}$, sends $x_{i}$ to $t^{u_{i}} x_{i}$ and $t$ to $t^{1-2 \epsilon}$, where $\epsilon=0,1$. So, we shall think $\mathbb{Z}^{n} \rtimes C_{2} \leqslant A u t(M)$.

Note that $A u t\left(F_{n}\right)$ and $\mathbb{Z}^{n} \rtimes C_{2}$ have trivial intersection as subgroups of $A u t(M)$. We shall now show that they generate $\operatorname{Aut}(M)$. Let $\Psi \in \operatorname{Aut}(M)$. It will be sufficient to show that we can multiply $\Psi$ by elements in $\operatorname{Aut}\left(F_{n}\right)$ and $\mathbb{Z}^{n} \rtimes C_{2}$ until we get the identity. Note that, since the centre of $M$ is its infinite cyclic subgroup generated by $t$ (use $n \geqslant 2$ and see Lemma 2.2), $\langle t\rangle$ is characteristic in $M$ and so, $t \Psi=t^{ \pm 1}$. Thus, after possibly composing with $v \in \mathbb{Z}^{n} \rtimes C_{2}$, we can assume that $t \Psi=t$. Then, write $x_{i} \Psi=t^{u_{i}} w_{i}$ for $i=1, \ldots, n$, and $u=\left(u_{1}, \ldots, u_{n}\right)^{T}$. Since $\Psi$ is an automorphism, $\left\{w_{1}, \ldots, w_{n}\right\}$ must generate (and so form a basis of) $F_{n}$. Composing $\Psi$ with the automorphism which fixes $t$ and sends $w_{i}$ back to $x_{i}, i=1, \ldots, n$, we obtain the element $\left(v^{0}, u\right) \in \mathbb{Z}^{n} \rtimes C_{2}$. This proves that $A u t\left(F_{n}\right)$ together with $\mathbb{Z}^{n} \rtimes C_{2}$ generate $A u t(M)$.

Consider now $\Theta \in \operatorname{Aut}\left(F_{n}\right)$ and $\left(v^{\epsilon}, u\right) \in \mathbb{Z}^{n} \rtimes C_{2}, u=\left(u_{1}, \ldots, u_{n}\right)^{T}$, viewed as elements of $\operatorname{Aut}(M)$. We shall calculate the conjugate $\Theta\left(v^{\epsilon}, u\right) \Theta^{-1}$ as an element of $A u t(M)$. Let $\theta=$ $\left.\Theta\right|_{F_{n}} \in \operatorname{Aut}\left(F_{n}\right)$ and let $\theta^{\mathrm{ab}}=\left(b_{i, j}\right) \in G L_{n}(\mathbb{Z})$ be its abelianisation (an $n \times n$ integral matrix whose $i$-th row describes the total exponent sums of $\left.x_{i} \theta\right)$. Bearing this in mind, $\Theta\left(v^{\epsilon}, u\right) \Theta^{-1}$ acts as

$$
\begin{aligned}
& x_{i} \mapsto x_{i} \Theta \mapsto t^{c_{i}}\left(x_{i} \Theta\right) \mapsto t^{c_{i}} x_{i} \\
& t \mapsto t \mapsto t^{1-2 \epsilon} \quad \mapsto t^{1-2 \epsilon},
\end{aligned}
$$


where $c_{i}=\sum_{j=1}^{n} b_{i, j} u_{j}$ is the $i$-th entry of the column vector $\theta^{\mathrm{ab}} u$. In other words,

$$
\Theta\left(v^{\epsilon}, u\right) \Theta^{-1}=\left(v^{\epsilon}, \theta^{\mathrm{ab}} u\right)
$$

for every $u=\left(u_{1}, \ldots, u_{n}\right)^{T} \in \mathbb{Z}^{n}$. This immediately shows that $\mathbb{Z}^{n} \rtimes C_{2}$ is normal in $A u t(M)$. Hence, $\operatorname{Aut}\left(F_{n} \times \mathbb{Z}\right) \cong\left(\mathbb{Z}^{n} \rtimes C_{2}\right) \rtimes \operatorname{Aut}\left(F_{n}\right)$, where the action of $\operatorname{Aut}\left(F_{n}\right)$ in this last semi-direct product is the trivial one over the $C_{2}$ part, and the natural one after abelianisation over the $\mathbb{Z}^{n}$ part.

Lastly, to prove the final statement note that, since $\langle t\rangle$ is central, inner automorphisms of $F_{n} \times \mathbb{Z}$ are just inner automorphisms by elements of $F_{n}$, and all of them fix $t$. Thus, $\operatorname{Inn}(M)=$ $\operatorname{Inn}\left(F_{n}\right) \leqslant \operatorname{Aut}\left(F_{n}\right)$ and so $\operatorname{Out}(M) \cong\left(\mathbb{Z}^{n} \rtimes C_{2}\right) \rtimes \operatorname{Out}\left(F_{n}\right)$, where the actions are just as before but factorised by $\operatorname{Inn}\left(F_{n}\right)$.

\section{$3 \quad \operatorname{Aut}\left(F_{2}\right)$ and $G L_{2}(\mathbb{Z})$}

For the rest of the paper we will be considering only the case $n=2$. Hence, we shall avoid unnecessary subscripts by using the letters $\{a, b\}$ as free generators of $F_{2}=\langle a, b\rangle$.

In this section we will briefly review some well known facts about $\operatorname{Aut}\left(F_{2}\right), \operatorname{Out}\left(F_{2}\right)$ and $G L_{2}(\mathbb{Z})$. The abelianisation map $F_{2} \rightarrow F_{2}^{\text {ab }}$ induces naturally a surjective map $\operatorname{Aut}\left(F_{2}\right) \rightarrow G L_{2}(\mathbb{Z})$ for which, abusing notation, we shall write $\phi \mapsto \phi^{\mathrm{ab}}$. More precisely, $\phi^{\mathrm{ab}}$ is the $2 \times 2$ integral matrix whose first (second) row counts the total $a$ - and $b$-exponent sums of $a \phi$ (of $b \phi$ ). Clearly, this is well defined for any rank, but in rank 2 it has some special properties which make this case easier to study; the most important of these is the following well known result (see [6, chapter 4, proposition 4.5]).

Theorem 3.1 (Nielsen) The kernel of the abelianisation from Aut $\left(F_{2}\right)$ to $G L_{2}(\mathbb{Z})$ consists of precisely the inner automorphisms of $F_{2}$. That is, Out $\left(F_{2}\right) \cong G L_{2}(\mathbb{Z})$.

This means that, for every automorphism $\phi \in A u t\left(F_{2}\right)$, the $2 \times 2$ integral matrix $\phi^{\text {ab }}$ is enough to recover the automorphism $\phi$ up to conjugation. Since the isomorphism type of $M_{\phi}$ (and so that of $A u t\left(M_{\phi}\right)$ ) only depends on $\phi$ up to conjugation, $\phi^{\text {ab }}$ contains all the algebraic information we may want about $M_{\phi}$ and $\operatorname{Aut}\left(M_{\phi}\right)$.

Matrices in $G L_{2}(\mathbb{Z})$ can be classified according to their eigenvalues and dynamics, often leading to useful information for $\operatorname{Aut}\left(F_{2}\right)$. This is often done in the following way.

Definition 3.2 Let $A$ be a $2 \times 2$ integral invertible matrix, $A \in G L_{2}(\mathbb{Z})$. If $A^{2}$ is the identity matrix, $A^{2}=I_{2}$, we say that $A$ is elliptic. Otherwise, $A$ is called hyperbolic if $\left|\operatorname{trace}\left(A^{2}\right)\right|>2$, parabolic if $\left|\operatorname{trace}\left(A^{2}\right)\right|=2$, and elliptic if $\left|\operatorname{trace}\left(A^{2}\right)\right|<2$.

Suppose $A \in G L_{2}(\mathbb{Z})$ is a hyperbolic matrix. Then, $A^{2} \neq I_{2}$ has two real eigenvalues, $\alpha, 1 / \alpha$, such that $|\alpha|>1$. Since $A$ preserves the one dimensional eigenspaces of $A^{2}, A$ must also have two real eigenvalues, $\beta, \pm 1 / \beta$, such that $|\beta|>1$. In particular, $A$ does not have 1 as an eigenvalue.

Suppose $A \in G L_{2}(\mathbb{Z})$ is a parabolic matrix. Then, $A^{2} \neq I_{2}$ has characteristic polynomial equal to $(x \pm 1)^{2}$. This implies that $A^{2}$ is conjugate, in $G L_{2}(\mathbb{Z})$, to $\pm\left(\begin{array}{ll}1 & k^{\prime} \\ 0 & 1\end{array}\right)$ for some $0 \neq k^{\prime} \in \mathbb{Z}$ (take a rational eigenvector of eigenvalue \pm 1 , multiply it by a scalar to obtain an integral vector $v$ 
with coprime entries, and then extend to a basis $\{u, v\}$ of $\left.\mathbb{Z}^{2}\right)$. A simple calculation shows now that $A$ must then be conjugate to one of the matrices

$$
\left(\begin{array}{ll}
1 & k \\
0 & 1
\end{array}\right) \text { or }\left(\begin{array}{rr}
-1 & k \\
0 & -1
\end{array}\right)
$$

for some integer $k \neq 0$. These are infinite order matrices and the first of these will turn out to be the most challenging case to consider.

Finally, suppose $A \in G L_{2}(\mathbb{Z})$ is an elliptic matrix. Then, either $A^{2}=I_{2}$ or the characteristic polynomial of $A^{2}$ is equal to $x^{2}+1, x^{2}+x+1$ or $x^{2}-x+1$. In the first case, $A$ is either $\pm I_{2}$ or is conjugate, in $G L_{2}(\mathbb{Z})$, to $\left(\begin{array}{cc}1 & k \\ 0 & -1\end{array}\right)$ for some $k \in \mathbb{Z}$ (by similar reasoning to that above). Otherwise, $A$ has complex conjugate roots and, in particular, it does not have 1 as an eigenvalue.

\section{The rank 2 case: proof of Theorem 1.1}

Given $\phi \in A u t\left(F_{2}\right)$, we shall analyse $A u t\left(M_{\phi}\right)$ and prove Theorem 1.1 by following the classification of matrices in the previous section for $\phi^{\mathrm{ab}}$.

First, we state the following two lemmas for later use.

Lemma 4.1 Every non-central element in $G L_{2}(\mathbb{Z})$ generates a finite index subgroup of its own centraliser. The centre consists precisely of the matrices $\pm I_{2}$.

Proof. The first statement of the lemma follows easily from the presentation of $G L_{2}(\mathbb{Z})$ as an amalgamated product, $G L_{2}(\mathbb{Z}) \cong D_{4} *_{D_{2}} D_{6}$, where $D_{n}$ is the dihedral group of order $2 n$. The statement about central elements is elementary.

Lemma 4.2 Consider the automorphism $\phi$ of $F_{2}=\langle a, b\rangle$ given by $a \phi=a b^{k}$ and $b \phi=b$, where $k \neq 0$. Then, for every integer $r \neq 0$ and every $w \in F_{2}$,

i) $F i x \phi=F i x \phi^{r}=\left\langle a b a^{-1}, b\right\rangle$,

ii) if $w \phi^{r}$ is conjugate to $w$, then $w$ is conjugate to an element fixed by $\phi$.

Proof. Given an arbitrary reduced word $w \in F_{2}$, let us split it into pieces each of the form $b^{m}, a b^{m}, b^{m} a^{-1}$ or $a b^{m} a^{-1}$, where $m$ is some integer. There can be many variants of such a splitting, but we shall use the special one defined by putting breaking points precisely before each occurrence of $a$ and after each occurrence of $a^{-1}$. One can easily see that this splitting is invariant under the action of $\phi$, and that the pieces do not interact under iterates of $\phi$. Hence, if $w \phi^{r}=w$ then the corresponding pieces in the splitting of $w$ must also be fixed by $\phi^{r}$, which rules out the possibilities $a b^{m}$ and $b^{m} a^{-1}$ (because $r k \neq 0$ ). This proves that Fix $\phi^{r} \leqslant\left\langle a b a^{-1}, b\right\rangle$. The inclusions $\left\langle a b a^{-1}, b\right\rangle \leqslant F i x \phi \leqslant F i x \phi^{r}$ are obvious. This proves (i).

In order to prove (ii), note that we can assume $w$ is cyclically reduced. If $w$ is a power of $b$ there is nothing to prove. So, we may also assume that $w$ contains $a^{ \pm 1}$. Moreover, by inverting and cyclically permuting if necessary, we may assume that $w$ begins with $a$. So, in this particular situation, assume that $w \phi^{r}$ is conjugate to $w$. The splitting of $w$ must begin with a piece of the form $a b^{m}$ or $a b^{m} a^{-1}$, and must end with a piece of the form $b^{m}$ or $a b^{m}$. But this splitting is stable under iterates of $\phi$ hence, the first and last pieces in $w \phi^{r}$ will be of the corresponding same 
types. In particular, $w \phi^{r}$ is still cyclically reduced. Thus, $w \phi^{r}$ must be a cyclic permutation of $w$. Then, for a suitable $s, w \phi^{r s}=w$ which, by (i), means that $w$ is fixed by $\phi$. This completes the proof. $\square$

Proof of Theorem 1.1. Throughout the proof, let us fix the following notation. Let $F_{2}=\langle a, b\rangle$ be a free group of rank $n=2$, let $\phi \in \operatorname{Aut}\left(F_{2}\right)$ and let $M_{\phi}=F_{2} \rtimes_{\phi} \mathbb{Z}$ be the mapping torus of $\phi$.

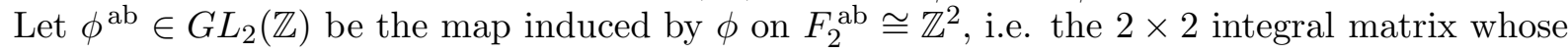
rows count the total $a$ - and $b$-exponent sums of $\{a \phi, b \phi\}$.

First of all, note that the discussions in the previous section show that a generic matrix $\phi^{\mathrm{ab}} \in$ $G L_{2}(\mathbb{Z})$ fits into one of the cases distinguished in Theorem 1.1. Namely, if $A$ is hyperbolic then it satisfies (iii), if it is parabolic it satisfies either (iii) or (v), and if it is elliptic then it fits into either (i), (ii) or (iv). Uniqueness is a straightforward exercise in linear algebra.

Suppose $\phi^{\mathrm{ab}}=I_{2}$. Then, $\phi=\gamma_{w}$ for some $w \in F_{2}$. Hence, using Lemma 2.1, $M_{\phi} \cong M_{I d}=$ $F_{2} \times \mathbb{Z}$. Now, using Theorem 2.5, we have

$$
\operatorname{Out}\left(M_{\phi}\right) \cong \operatorname{Out}\left(M_{I d}\right) \cong\left(\mathbb{Z}^{2} \rtimes C_{2}\right) \rtimes G L_{2}(\mathbb{Z}),
$$

where the actions are the natural ones described above. This proves Theorem 1.1 (i).

Suppose $\phi^{\mathrm{ab}}=-I_{2}$. Then it does not have 1 as an eigenvalue. Hence, by Theorem 2.4 $\mathrm{Out}^{+}\left(M_{\phi}\right)$ is a normal subgroup of $\operatorname{Out}\left(M_{\phi}\right)$ of index at most two which is isomorphic to $C\left(\phi^{\mathrm{ab}}\right) /\left\langle\phi^{\mathrm{ab}}\right\rangle$, where $C\left(\phi^{\mathrm{ab}}\right)$ is the centraliser of $\phi^{\mathrm{ab}}$ in $\operatorname{Out}\left(F_{2}\right)=G L_{2}(\mathbb{Z})$. But $\phi^{\mathrm{ab}}=-I_{2}$, which is central in $G L_{2}(\mathbb{Z})$ so,

$$
\operatorname{Out}^{+}\left(M_{\phi}\right) \cong C\left(\phi^{\mathrm{ab}}\right) /\left\langle\phi^{\mathrm{ab}}\right\rangle=G L_{2}(\mathbb{Z}) /\left\{ \pm I_{2}\right\}=P G L_{2}(\mathbb{Z}) .
$$

On the other hand, $a \mapsto a, b \mapsto b, t \mapsto t^{-1}$ determines a (well-defined) negative automorphism $\Upsilon$ of $M_{\phi}$ and so, $A u t^{+}\left(M_{\phi}\right) \unlhd_{2} \operatorname{Aut}\left(M_{\phi}\right)$. Furthermore, note that $\Upsilon$ has order two and commutes with every $\Psi \in \operatorname{Aut}^{+}\left(M_{\phi}\right)$ (which has the form $a \mapsto w_{1}, b \mapsto w_{2}, t \mapsto t w_{3}$ where $w_{3}$ is a palindrome, $\left.w_{3}^{R}=w_{3}\right)$. Hence, $\operatorname{Aut}\left(M_{\phi}\right) \cong A u t^{+}\left(M_{\phi}\right) \times C_{2}$. Finally, since $\operatorname{Inn}\left(M_{\phi}\right) \leqslant$ $\operatorname{Aut}^{+}\left(M_{\phi}\right)$, we have

$$
\operatorname{Out}\left(M_{\phi}\right) \cong \operatorname{Out}^{+}\left(M_{\phi}\right) \times C_{2} \cong P G L_{2}(\mathbb{Z}) \times C_{2} .
$$

This proves Theorem 1.1 (ii).

Suppose that $\phi^{\mathrm{ab}} \neq-I_{2}$ does not have 1 as an eigenvalue. Then, applying Theorem 2.4 and Lemma 4.1] we deduce that $\operatorname{Out}\left(M_{\phi}\right)$ is finite. This proves Theorem 1.1 (iii).

Now, suppose that $\phi^{\text {ab }}$ is conjugate in $G L_{2}(\mathbb{Z})$ to $\left(\begin{array}{cc}1 & k \\ 0 & -1\end{array}\right)$ for some $k \in \mathbb{Z}$. Then, Proposition 4.3 shows that $\operatorname{Out}\left(M_{\phi}\right)$ has an infinite cyclic subgroup of finite index. This completes Theorem 1.1 (iv).

Finally, suppose that $\phi^{\text {ab }}$ is conjugate in $G L_{2}(\mathbb{Z})$ to $\left(\begin{array}{ll}1 & k \\ 0 & 1\end{array}\right)$ for some $0 \neq k \in \mathbb{Z}$. Then, Proposition 4.4 will complete the proof of Theorem 1.1(v) by showing that $\operatorname{Out}\left(M_{\phi}\right)$ also has an infinite cyclic subgroup of finite index.

Proposition 4.3 With the notation above, assume $\phi^{\text {ab }}$ is conjugate in $G L_{2}(\mathbb{Z})$ to $\left(\begin{array}{cc}1 & k \\ 0 & -1\end{array}\right)$, where $k \in \mathbb{Z}$. Then Out $\left(M_{\phi}\right)$ has an infinite cyclic subgroup of finite index. 
Proof. Using Lemma 2.1, we can assume $\phi^{\mathrm{ab}}=\left(\begin{array}{cc}1 & k \\ 0 & -1\end{array}\right)$. Furthermore, composing $\phi$ by a suitable inner automorphism of $F_{2}$, we can assume $a \phi=a b^{k}$ and $b \phi=b^{-1}$. So,

$$
M_{\phi}=\left\langle a, b, t \mid t^{-1} a t=a b^{k}, t^{-1} b t=b^{-1}\right\rangle \text {. }
$$

It is straightforward to check that

$$
\begin{aligned}
a & \mapsto t a \\
b & \mapsto b \\
t & \mapsto t .
\end{aligned}
$$

defines an automorphism $\Psi$ of $M_{\phi}$ such that $[\Psi] \in \operatorname{Out}\left(M_{\phi}\right)$ is an infinite order outer automorphism (because inner automorphisms of $M_{\phi}$ leave $F_{2}$ invariant). Let us prove now that the infinite cyclic subgroup $\langle[\Psi]\rangle$ has finite index in $\operatorname{Out}\left(M_{\phi}\right)$.

Clearly, $\phi^{2}=I d$ and so, $t^{2}$ is in the centre of $M_{\phi}$. Also, $\left\langle a, b, t^{2}\right\rangle \cong F_{2} \times \mathbb{Z}$ is an index 2 subgroup of $M_{\phi}$. So, all those automorphisms of $M_{\phi}$ which restrict to an automorphism of $\left\langle a, b, t^{2}\right\rangle$ form a finite index subgroup of $A u t\left(M_{\phi}\right)$ (since $M_{\phi}$ is finitely generated and so has finitely many index 2 subgroups). Moreover, the centre of $\left\langle a, b, t^{2}\right\rangle$ is $\left\langle t^{2}\right\rangle$ so, any such automorphism sends $t^{2}$ to $t^{ \pm 2}$. Hence, all those automorphisms of $M_{\phi}$ which restrict to an automorphism of $\left\langle a, b, t^{2}\right\rangle$ and fix $t^{2}$ still form a finite index subgroup of $\operatorname{Aut}\left(M_{\phi}\right)$, containing $\Psi^{2}$. Thus, we can confine our attention to the subgroup $G \leqslant A u t\left(M_{\phi}\right)$ consisting on these automorphisms, and prove that $\left\langle\left[\Psi^{2}\right]\right\rangle$ has finite index in $[G] \leqslant \operatorname{Out}\left(M_{\phi}\right)$.

Let $\Theta \in G$. Note that $[a, t]=a^{-1} t^{-1} a t=b^{k}$ and $[b, t]=b^{-1} t^{-1} b t=b^{-2}$ and hence $M_{\phi}^{\prime}=\left\langle b^{2}\right\rangle F_{2}^{\prime}$ if $k$ is even, and $M_{\phi}^{\prime}=\langle b\rangle F_{2}^{\prime}$ if $k$ is odd. Also, $M_{\phi}^{\text {ab }}$ has torsion subgroup generated by $b$ if $k$ is even, and is torsion-free if $k$ is odd. In any case, the preimage in $M_{\phi}$ of the (possibly trivial) torsion in $M_{\phi}^{\mathrm{ab}}$ is $\langle b\rangle M_{\phi}^{\prime}=\langle b\rangle F_{2}^{\prime}$. In particular, this subgroup is characteristic in $M_{\phi}$ and so $\Theta$ acts on $\left\langle a, b, t^{2}\right\rangle$ in the following way:

$$
\begin{aligned}
a & \mapsto t^{r} u \\
b & \mapsto v \\
t^{2} & \mapsto t^{2}
\end{aligned}
$$

where $u \in F_{2}, v \in\langle b\rangle F_{2}^{\prime}$, and $r$ is even. Since $\left\langle a, b, t^{2}\right\rangle=\left\langle t^{r} u, v, t^{2}\right\rangle=\left\langle u, v, t^{2}\right\rangle$ and $t^{2}$ lies in the centre of $M_{\phi}$, it follows that $\langle u, v\rangle=\langle a, b\rangle$. Then, from this and the form of $v$, we deduce that $u \in a^{\epsilon}\langle b\rangle F_{2}^{\prime}$ for some $\epsilon= \pm 1$.

Consider now the automorphism $\Lambda=\Theta \Psi^{-\epsilon r}$ which acts like

$$
\begin{aligned}
& a \mapsto t^{r} u \mapsto t^{r}\left(u \Psi^{-\epsilon r}\right)=x \in F_{2} \\
& b \mapsto v \mapsto v \Psi^{-\epsilon r}=y \in F_{2} \\
& t^{2} \mapsto t^{2} \mapsto t^{2}
\end{aligned}
$$

(note that $u$ has $a$-exponent sum equal to $\epsilon$ and so, the $t$-exponent sum of $u \Psi^{-\epsilon r}$ is $-r$, showing that $x \in F_{2}$; also, the $a$-exponent sum of $v$ is 0 and so $y \in F_{2}$ too). Writing $t \Lambda=t^{s} w$ and imposing $t^{2}$ to be fixed, we deduce $t \Lambda=t z$ for some $z \in F_{2}$. Thus, $\Lambda$ is a positive automorphism of $M_{\phi}$ and, by Proposition [2.3, $\left(\left.\Lambda\right|_{F_{2}}\right)^{\text {ab }}$ lies in the centraliser of $\phi^{\mathrm{ab}}$. But a straightforward matrix calculation shows that $C\left(\phi^{\mathrm{ab}}\right)$ is finite and so, $(x, y)$ takes only finitely many values up to conjugacy in $F_{2}$. Since, by Proposition $2.3, z$ is uniquely determined by $(x, y), \Lambda$ also takes only finitely many values up to conjugacy, while $\Theta$ runs over all $G$. In other words, $\left\langle\left[\Psi^{2}\right]\right\rangle$ has finite index in $[G]$.

Proposition 4.4 With the notation above, assume $\phi^{\text {ab }}$ is conjugate in $G L_{2}(\mathbb{Z})$ to $\left(\begin{array}{ll}1 & k \\ 0 & 1\end{array}\right)$, where $0 \neq k \in \mathbb{Z}$. Then $\operatorname{Out}\left(M_{\phi}\right)$ has an infinite cyclic subgroup of finite index. 
Proof. Using Lemma 2.1, we can assume $\phi^{\mathrm{ab}}=\left(\begin{array}{cc}1 & k \\ 0 & 1\end{array}\right)$ and, furthermore, composing $\phi$ by a suitable inner automorphism of $F_{2}$, we can assume $a \phi=a b^{k}$ and $b \phi=b, k \neq 0$. So, we have to understand the automorphism group of the group

$$
M_{\phi}=\left\langle a, b, t \mid t^{-1} a t=a b^{k}, t^{-1} b t=b\right\rangle .
$$

Before going into the analysis of $A u t\left(M_{\phi}\right)$, note that both relators have $a$ - and $t$-exponent sums equal to zero. So, it makes sense to talk about $a$ - and $t$-exponent sums of elements in $M_{\phi}$. Strictly speaking, the maps from $M_{\phi}$ to $\mathbb{Z}$ killing $b$ and $t$ and sending $a$ to the generator of $\mathbb{Z}$ (resp. killing $a$ and $b$ and sending $t$ to the generator of $\mathbb{Z}$ ) are well defined surjective homomorphisms. They count the total $a$ - and $t$-exponent sums, respectively (note that the notion of $b$-exponent sum in $M_{\phi}$ is only well defined modulo $k$ ).

It is straightforward to check that

$$
\begin{aligned}
a & \mapsto t a \\
b & \mapsto b \\
t & \mapsto t
\end{aligned}
$$

defines an automorphism $\Psi$ of $M_{\phi}$ such that $[\Psi] \in \operatorname{Out}\left(M_{\phi}\right)$ is an infinite order outer automorphism (because inner automorphisms of $M_{\phi}$ leave $F_{2}$ invariant). Let us prove now that the infinite cyclic subgroup $\langle[\Psi]\rangle$ has finite index in $\operatorname{Out}\left(M_{\phi}\right)$.

Consider the three automorphisms of $M_{\phi}$ defined on the generators by

$\begin{array}{rlrlll}\Omega & & & \Delta & & \Xi \\ a & \mapsto b^{2} & a & \mapsto a^{-1} & a & \mapsto a b \\ b & \mapsto b^{-1} & b & \mapsto b^{-1} & b & \mapsto b \\ t & \mapsto t^{-1} & t & \mapsto t b^{-k} & t & \mapsto b t\end{array}$

(as above, checking that they are well-defined is a straightforward exercise).

Claim: for any given $\Theta \in \operatorname{Aut}\left(M_{\phi}\right)$, there exists an integer $m$ and an element $g \in M_{\phi}$ such that $\Theta \Psi^{m} \Gamma_{g}$ is equal to one of $\Xi^{i}, \Xi^{i} \Omega, \Xi^{i} \Delta$ or $\Xi^{i} \Delta \Omega$, for some $0 \leqslant i \leqslant|k|-1$.

This automatically will imply that $\langle[\Psi]\rangle$ has finite index in $\operatorname{Out}\left(M_{\phi}\right)$. In order to prove this claim note that, since $\operatorname{Inn}\left(M_{\phi}\right)$ is a normal subgroup of $\operatorname{Aut}\left(M_{\phi}\right)$, we may apply inner automorphisms at any point in the product $\Theta \Psi^{m}$.

So, let $\Theta$ be an arbitrary automorphism of $M_{\phi}$, and write normal forms for the images of generators, $a \Theta=t^{p} w_{1}, b \Theta=t^{l} w_{2}^{\prime}, t \Theta=t^{q} w_{3}$, where $w_{1}, w_{2}^{\prime}, w_{3} \in F_{2}$, and $p, l, q \in \mathbb{Z}$. Write also $w_{2}^{\prime}=w_{2}^{r}$, where $r \geqslant 1$ and $w_{2}$ is either trivial or not a proper power. Applying $\Theta$ to the equality $t^{-1} a t=a b^{k}$ we get

$$
w_{3}^{-1} t^{-q} t^{p} w_{1} t^{q} w_{3}=t^{p} w_{1}\left(t^{l} w_{2}^{\prime}\right)^{k} .
$$

Comparing the $t$-exponent sums we immediately see that $k l=0$ and hence $l=0$ and $w_{2} \neq 1$. Now, applying $\Theta$ to $t^{-1} b t=b$, we also get

$$
w_{3}^{-1}\left(w_{2} \phi^{q}\right)^{r} w_{3}=w_{3}^{-1} t^{-q} w_{2}^{\prime} t^{q} w_{3}=w_{2}^{\prime}=w_{2}^{r} .
$$

Thus, $w_{2} \phi^{q}$ is conjugate to $w_{2}$ in $F_{2}$. By applying Lemma 4.2 (ii), we obtain that $w_{2}$ is conjugate to an element fixed by $\phi$, say $w_{2}=x v_{2} x^{-1}$, where $x \in F_{2}$ and $1 \neq v_{2}=v_{2} \phi$ is not a proper power. Now, $\Theta \Gamma_{x}$ is an automorphism of $M_{\phi}$ acting as

$$
\begin{aligned}
& a \mapsto t^{p} w_{1} \mapsto x^{-1} t^{p} w_{1} x=t^{p} v_{1} \\
& b \mapsto w_{2}^{\prime} \mapsto x^{-1} w_{2}^{r} x=v_{2}^{r} \\
& t \mapsto t^{q} w_{3} \mapsto x^{-1} t^{q} w_{3} x=t^{q} v_{3},
\end{aligned}
$$


where $v_{1}, v_{3} \in F_{2}$. Since $b$ commutes with $t, v_{2}^{r}$ must commute with $t^{q} v_{3}$. But $v_{2}$ commutes with $t$ since it is fixed by $\phi$. Therefore $v_{2}$ commutes with $v_{3}$ and hence, $v_{3}=v_{2}^{s}$ for some integer $s$. Finally, observe that $\left\{t^{p} v_{1}, v_{2}^{r}, t^{q} v_{2}^{s}\right\}$ must generate $M_{\phi}$. Thus, since the $a$-exponent sum of $v_{2}$ is zero (by Lemma4.2 (i)), the $a$-exponent sum of $v_{1}$ must be \pm 1 . So, without loss of generality, we may assume that $\Theta$ acts as

$$
\begin{aligned}
a & \mapsto t^{p} v_{1} \\
b & \mapsto v_{2}^{r} \\
t & \mapsto t^{q} v_{2}^{s},
\end{aligned}
$$

where $v_{1}, v_{2} \in F_{2}, p, q, r, s \in \mathbb{Z}, v_{2}$ is fixed by $\phi$ and has $a$-exponent sum equal to zero, and $v_{1}$ has $a$-exponent sum equal to $\epsilon= \pm 1$.

Now let $m=-\epsilon p$. It is straightforward to verify that $\Theta \Psi^{m}$ acts in the following form,

$$
\begin{aligned}
& a \mapsto t^{p} v_{1} \mapsto t^{p}\left(v_{1} \Psi^{m}\right)=u_{1} \in F_{2} \\
& b \mapsto v_{2}^{r} \mapsto u_{2} \in F_{2} \\
& t \mapsto t^{q} v_{2}^{s} \mapsto t^{q} u_{3},
\end{aligned}
$$

where $u_{1}, u_{2}, u_{3} \in F_{2}$. By Proposition 2.3 it follows now that $\Theta \Psi^{m}$ restricts to an automorphism of $F_{2}$ with signum $q= \pm 1$.

Consider now the automorphisms $\Theta \Psi^{m}, \Theta \Psi^{m} \Omega, \Theta \Psi^{m} \Delta$ and $\Theta \Psi^{m} \Omega \Delta$. Each of these leaves $F_{2}$ invariant and have signum $q,-q, q$ and $-q$, respectively. Also, the traces of the abelianisations of their restrictions to $F_{2}$ are $d, e,-d$ and $-e$, respectively, for some $d, e \in \mathbb{Z}$. So, one of these four automorphisms, say $\Upsilon$, is positive and its restriction to $F_{2}$ abelianises to a matrix with non-negative trace.

We shall show that, up to an inner automorphism, $\Upsilon$ coincides with $\Xi^{i}$ for some $0 \leqslant i \leqslant|k|-1$. This will prove the claim since both $\Omega$ and $\Delta$ above have order two in $\operatorname{Aut}\left(M_{\phi}\right)$.

Since $\Upsilon$ is a positive automorphism, Proposition 2.3 ensures us that the matrices $\left(\left.\Upsilon\right|_{F_{2}}\right)^{\text {ab }}$ and $\phi^{\mathrm{ab}}=\left(\begin{array}{ll}1 & k \\ 0 & 1\end{array}\right)$ do commute. But the centraliser of $\phi^{\mathrm{ab}}$ in $G L_{2}(\mathbb{Z})$ is the set of matrices of the form $\left(\begin{array}{cc}1 & * \\ 0 & 1\end{array}\right)$ or $\left(\begin{array}{cc}-1 & * \\ 0 & -1\end{array}\right)$. So, since $\left(\left.\Upsilon\right|_{F_{2}}\right)$ ab has non-negative trace, we deduce that, for some $z \in F_{2}$, $\left.\left(\Upsilon \Gamma_{z}\right)\right|_{F_{2}}$ acts as $a \mapsto a b^{j}, b \mapsto b$, for some integer $j$. Write $j=i+\lambda k$ with $0 \leqslant i \leqslant|k|-1$, and notice that the inner automorphism $\Gamma_{t}$ of $M_{\phi}$ acts as

$$
\begin{aligned}
a & \mapsto a b^{k} \\
b & \mapsto b \\
t & \mapsto t .
\end{aligned}
$$

Hence $\Upsilon \Gamma_{z} \Gamma_{t}^{-\lambda}$ agrees with $\Xi^{i}$ on $F_{2}$. And they both are positive so, by Proposition [2.3 (ii), they must also agree on $t$. Hence, up to a conjugation, $\Upsilon$ coincides with $\Xi^{i}$. This completes the proof.

\section{Acknowledgments}

The first named author is partially supported by the grant of the President of Russian Federation for young Doctors MD-326.2003.01, by the INTAS grant N 03-51-3663, and by the Centre de Recerca Matemàtica (CRM) at Barcelona. The second named author gratefully acknowledges the support of the CRM and the UPC. The third named author is partially supported by DGI (Spain) through grant BFM2003-06613, and by the Generalitat de Catalunya through grant ACI-013. The three authors thank the CRM for their hospitality during the academic course 2004-2005, while this paper has been written. 


\section{References}

[1] Bestvina M. and Feighn M., Stable actions of groups on real trees, Invent. Math., 121, n. 2 (1995), 287-321.

[2] Bestvina M., Feighn M. and Handel M., The Tits alternative for Out $\left(F_{n}\right)$ I: Dynamics of exponentially growing automorphisms, Annals of Math., 151 (2000), 517-623.

[3] Brinkmann P., Splittings of mapping tori of free group automorphisms, Geometriae Dedicata, 93 (2002), 191-203.

[4] Ghys E., Sergiescu V., Stabilité et conjugaison differentiable pour certains feuilletages, Topology, 19 (1979), 179-197.

[5] B. Herrera, Sobre la multiestructura transversa de las foliaciones de Lie, tesina U.A.B. 173 (1993).

[6] Lyndon, R. and Schupp, P. Combinatorial group theory, Springer-Verlag, Berlin-New York, 1977. 